\title{
Physics-Based Elastic Image Registration Using Splines and Including Landmark Localization Uncertainties
}

\author{
Stefan Wörz and Karl Rohr \\ University of Heidelberg, IPMB, and DKFZ Heidelberg, \\ Dept. Bioinformatics and Functional Genomics, Biomedical Computer Vision Group \\ Im Neuenheimer Feld 364, 69120 Heidelberg, Germany \\ s.woerz@dkfz.de
}

\begin{abstract}
We introduce an elastic registration approach which is based on a physical deformation model and uses Gaussian elastic body splines (GEBS). We formulate an extended energy functional related to the Navier equation under Gaussian forces which also includes landmark localization uncertainties. These uncertainties are characterized by weight matrices representing anisotropic errors. Since the approach is based on a physical deformation model, cross-effects in elastic deformations can be taken into account. Moreover, we have a free parameter to control the locality of the transformation for improved registration of local geometric image differences. We demonstrate the applicability of our scheme based on 3D CT images from the Truth Cube experiment, 2D MR images of the brain, as well as $2 \mathrm{D}$ gel electrophoresis images. It turns out that the new scheme achieves more accurate results compared to previous approaches.
\end{abstract}

\section{Introduction}

The registration of $2 \mathrm{D}$ and $3 \mathrm{D}$ biomedical images is an important task, however, it is difficult and challenging. One reason is that in many applications it is still not quite clear which type of image information is optimal for matching. Another reason is that the spectrum of possible geometric differences is relatively large.

Generally, nonrigid schemes are required for image registration (for a recent survey see [1]). A special class of nonrigid transformations are elastic transformations, which allow to cope with local shape differences, and which are typically based on an energy functional or the related partial differential equation (PDE). To compute an elastic transformation, often spline-based approaches are used, which can be subdivided into schemes based on a uniform grid of control points, where typically B-splines are used, and schemes based on a nonuniform grid of control points (e.g., 23456/78910]). The latter type of spline-based schemes generally requires a smaller number of control points (landmarks). Examples of such schemes are based on thin-plate splines (TPS, e.g., 468), elastic body splines (EBS, e.g., [2]), and Gaussian EBS (GEBS, e.g., 5910]). TPS are based 

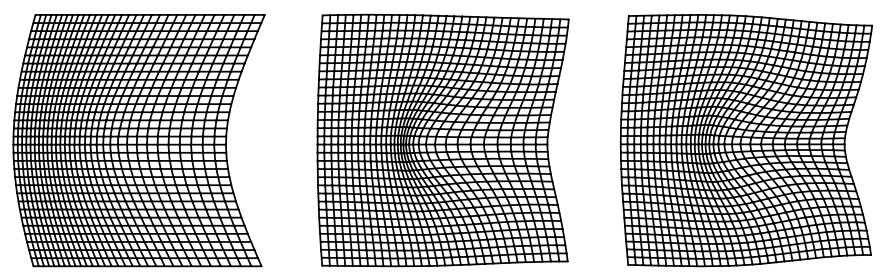

Fig. 1. Deformations obtained for the displacement of a single landmark using TPS (left) as well as GEBS: $\nu=0$ (middle) and $\nu=0.49$ (right)

on the bending energy of a thin plate, which represents a relatively coarse deformation model. In comparison, EBS and GEBS are derived from the Navier equation, which describes the deformation of homogeneous elastic tissues (bodies). In spline-based registration approaches, generally an interpolation scheme is used that forces corresponding landmarks to exactly match each other. The underlying assumption is that the landmark positions are known exactly. In real applications, however, landmark localization is always prone to error. Therefore, to take into account landmark errors, approximation schemes have been proposed, for example, for TPS (e.g., 4]). However, approximation schemes for EBS and GEBS approaches have not yet been introduced.

We have developed a physics-based approach for elastic image registration based on GEBS. To incorporate localization uncertainties of landmarks we introduce an extended energy functional related to the Navier equation. By inclusion of weight matrices we can cope with anisotropic uncertainties, i.e. uncertainties that are different in different directions. Our scheme results from an analytic solution of the corresponding extended Navier equation using Gaussian forces. Note that the derivation of our scheme significantly differs from the derivation of approximating TPS. The reason is that the underlying PDEs of GEBS and TPS as well as the assumed image forces (i.e. Gaussian forces and Dirac forces, respectively) are different. Central to our scheme is that it includes a material parameter (Poisson ratio $\nu$ ), which defines the ratio between transverse contraction and longitudinal dilation of an elastic material. Therefore, cross-effects can be taken into account which is not the case for TPS. Fig. 1 illustrates this effect for the case of a single landmark which is displaced horizontally (particularly note the vertical deformations in Fig. 1, right). Moreover, we have a free parameter to control the locality of the transformation.

\section{Gaussian Elastic Body Splines (GEBS)}

\subsection{Interpolating GEBS}

In this section, we briefly describe the interpolating GEBS approach [5]. This approach is based on the Navier equation of linear elasticity

$$
\widetilde{\mu} \Delta \mathbf{u}+(\widetilde{\lambda}+\widetilde{\mu}) \nabla(\operatorname{div} \mathbf{u})+\mathbf{f}=\mathbf{0}
$$


with the displacement vector field $\mathbf{u}$, body forces $\mathbf{f}$, as well as Lamé constants $\widetilde{\mu}, \widetilde{\lambda}>0$ describing material properties. Given Gaussian forces $\mathbf{f}(\mathbf{x})=\mathbf{c} f(r)=$ $\mathbf{c}\left(\sqrt{2 \pi} \sigma_{f}\right)^{-3} \exp \left(-\frac{r^{2}}{2 \sigma_{f}^{2}}\right)$ with $\mathbf{x}=(x, y, z)^{T}, r=\sqrt{x^{2}+y^{2}+z^{2}}$, and the standard deviation $\sigma_{f}$, an analytic solution of (1) can be derived [5]. The resulting matrix-valued basis function $\mathbf{G}$ (a $3 \times 3$ matrix) reads (up to a constant factor)

$$
\mathbf{G}(\mathbf{x})=\left(\frac{\alpha r^{2}+\sigma_{f}^{2}}{r^{3}} \operatorname{erf}(\hat{r})-\beta \frac{e^{-\hat{r}^{2}}}{r^{2}}\right) \mathbf{I}+\left(\frac{r^{2}-3 \sigma_{f}^{2}}{r^{5}} \operatorname{erf}(\hat{r})+3 \beta \frac{e^{-\hat{r}^{2}}}{r^{4}}\right) \mathbf{x x}^{T}
$$

where $\hat{r}=r /\left(\sqrt{2} \sigma_{f}\right), \alpha=3-4 \nu, \beta=\sigma_{f} \sqrt{2 / \pi}$, and the error function $\operatorname{erf}(x)=$ $2 \pi^{-1 / 2} \int_{0}^{x} e^{-\xi^{2}} d \xi$. I denotes the $3 \times 3$ identity matrix and $\nu$ is the Poisson ratio $\nu=\widetilde{\lambda} /(2 \widetilde{\lambda}+2 \widetilde{\mu}), 0 \leq \nu<0.5$. In contrast, in the original EBS approach [2] polynomial or rational forces are used. Using the interpolation condition $\mathbf{q}_{i}=$ $\mathbf{u}\left(\mathbf{p}_{i}\right)$, the scheme for spline-based elastic image registration is given by $\mathbf{u}(\mathbf{x})=$ $\mathbf{x}+\sum_{i=1}^{n} \mathbf{G}\left(\mathbf{x}-\mathbf{p}_{i}\right) \mathbf{c}_{i}$, where $\mathbf{p}_{i}$ and $\mathbf{q}_{i}(i=1, \ldots, n)$ denote the positions of the $n$ landmarks of the source and target image, respectively. The coefficients $\mathbf{c}_{i}$ represent the strength and direction of the Gaussian forces.

\subsection{Approximating GEBS}

With the interpolation approach described above the landmarks are matched exactly. This implicitly assumes that the landmark positions are known exactly. To take into account landmark localization errors we use the approximation condition $\mathbf{q}_{i} \approx \mathbf{u}\left(\mathbf{p}_{i}\right)$ and include $3 \times 3$ covariance matrices $\boldsymbol{\Sigma}_{i}$ defining the anisotropic localization uncertainties of the landmarks $i=1, \ldots, n$. To derive approximating GEBS, we introduce an energy-minimizing functional consisting of two terms. The first term $J_{\text {Elastic }}$ represents the elastic energy according to the Navier equation (11) without the force $\mathbf{f}$ and acts as a smoothness term. The corresponding Lagrange function is given by

$$
\begin{aligned}
L_{\text {Elastic }}= & \widetilde{\mu}\left(u_{x}^{2}+v_{y}^{2}+w_{z}^{2}\right)+\widetilde{\mu}\left(\left(u_{y}+v_{x}\right)^{2}+\left(u_{z}+w_{x}\right)^{2}+\left(v_{z}+w_{y}\right)^{2}\right) / 2 \\
& +\widetilde{\lambda}\left(u_{x}^{2}+v_{y}^{2}+w_{z}^{2}+2 u_{x} v_{y}+2 u_{x} w_{z}+2 v_{y} w_{z}\right) / 2,
\end{aligned}
$$

where $\mathbf{u}=(u, v, w)^{T}=\mathbf{u}(\mathbf{x})$ and the subscripts $x, y$, and $z$ denote first order partial derivatives. The second term $J_{\text {Force }}$ represents Gaussian forces specified by corresponding landmarks $\mathbf{p}_{i}$ and $\mathbf{q}_{i}$, and, in addition, incorporates the localization uncertainties $\boldsymbol{\Sigma}_{i}$ of the landmarks. Here, we propose a quadratic approximation $J_{\text {Force }}$, which is given by the following Lagrange function

$$
L_{\text {Force }}=\frac{1}{\lambda n} \sum_{i=1}^{n} f\left(\mathbf{x}-\mathbf{p}_{i}\right)\left(\mathbf{q}_{i}-\mathbf{u}(\mathbf{x})\right)^{T} \boldsymbol{\Sigma}_{i}^{-1}\left(\mathbf{q}_{i}-\mathbf{u}(\mathbf{x})\right)
$$

where $\lambda>0$ denotes the regularization parameter. Note that $L_{\text {Force }}$ includes Gaussian forces $f\left(\mathbf{x}-\mathbf{p}_{i}\right)$ in contrast to Dirac forces in the case of TPS. The combined functional $J_{\lambda}$ then reads 


$$
J_{\lambda}=J_{\text {Elastic }}+J_{\text {Force }}=\int_{-\infty}^{\infty} \int_{-\infty}^{\infty} \int_{-\infty}^{\infty}\left(L_{\text {Elastic }}(\mathbf{x}, \mathbf{u})+L_{\text {Force }}(\mathbf{x}, \mathbf{u})\right) d \mathbf{x}
$$

The corresponding PDE can be derived as

$$
\widetilde{\mu} \Delta \mathbf{u}+(\widetilde{\lambda}+\widetilde{\mu}) \nabla(\operatorname{div} \mathbf{u})+\nabla_{\mathbf{u}} L_{\text {Force }}=\mathbf{0},
$$

and represents an extension of the Navier equation (11). The solution to (6) can be determined in analytic form and it turns out that it is the same as in the case of interpolation, i.e. it consists of the same matrix-valued basis function $\mathbf{G}$. However, the resulting linear system of equations to compute the coefficients $\mathbf{c}_{i}$ differs. More precisely, the structure of the linear system of equations is the same for interpolating and approximating GEBS, except that approximating GEBS include additional sums of weighted forces $\sum_{i=1}^{n} f\left(\mathbf{p}_{j}-\mathbf{p}_{i}\right) \boldsymbol{\Sigma}_{i}^{-1}$.

\subsection{Means to Improve Stability and Efficiency}

To compute the matrix-valued basis function $\mathbf{G}$ in (2), we have to consider two different aspects. First, for small values of $r<0.001$ the computed values for $\mathbf{G}$ are numerically not stable, which is caused by fractions that involve very small numerators and denominators. However, this problem can easily be solved by employing the limit of $\mathbf{G}$ for $r \rightarrow 0$, which is given by

$$
\lim _{r \rightarrow 0} \mathbf{G}(\mathbf{x})=\frac{1}{16 \pi \sigma \widetilde{\mu}} \frac{1}{1-\nu} \sqrt{\frac{2}{\pi}}\left(\frac{10}{3}-4 \nu\right) \mathbf{I} .
$$

Second, G involves the computation of the Gaussian error function, which is a costly operation in comparison to, e.g., a multiplication. To reduce the computation time, we exploit the fact that for $r \gg \sigma$ the basis function $\mathbf{G}$ reduces to

$$
\mathbf{G}(\mathbf{x}) \approx \frac{1}{16 \pi \widetilde{\mu}} \frac{1}{1-\nu}\left(\frac{3-4 \nu}{r} \mathbf{I}+\frac{\mathbf{x x}^{T}}{r^{3}}\right)
$$

In our implementation, we use this approximation for $r>18 \sigma$ where the error is below $1 \%$, while the computation time is reduced by a factor of about 2 .

\section{Experimental Results}

\subsection{D CT Truth Cube Images}

To validate our scheme we have used 3D CT images from the Truth Cube experiment [11]. The Truth Cube is a silicone rubber cube, which comprises 343 regularly distributed small Teflon spheres (see Fig. 2, left). Using a compression plate, a force is applied to the top face yielding a compressed cube. Images have been acquired for the uncompressed cube as well as for three different compressions (nominal strains of $5 \%, 12.5 \%$, and $18.25 \%$ ). In each of the four 3D CT images, the positions of all 343 spheres have been determined. We have registered the $3 \mathrm{D}$ image of the uncompressed cube to each of the three compressed cube 

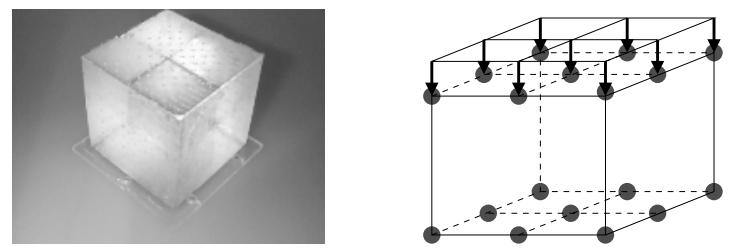

Fig. 2. Truth Cube with 343 small spheres (left, from [11]) and sketch of Truth Cube with nine landmarks at the bottom face and nine landmarks at the top face (right)

Table 1. Mean geometric error $\bar{e}_{\text {geom }}$ and mean intensity error $\bar{e}_{i n t}$ for the registration of 3D Truth Cube images as well as the improvement w.r.t. the unregistered case

\begin{tabular}{|l|l||c|c||c|c||c|c|}
\hline & \multicolumn{4}{|c||}{} & \multicolumn{2}{c||}{$5 \%$ strain } & \multicolumn{2}{c||}{$12.5 \%$ strain } & \multicolumn{2}{c|}{$18.25 \%$ strain } \\
& & $\bar{e}_{\text {geom }}$ & $\bar{e}_{\text {int }}$ & $\bar{e}_{\text {geom }}$ & $\bar{e}_{\text {int }}$ & $\bar{e}_{\text {geom }}$ & $\bar{e}_{\text {int }}$ \\
\hline \hline Unregistered & absolute & $2.6 \mathrm{~mm}$ & $16.9 \mathrm{gr}$ & $6.1 \mathrm{~mm}$ & $30.7 \mathrm{gr}$ & $9.2 \mathrm{~mm}$ & $41.2 \mathrm{gr}$ \\
\hline \hline Interpol. TPS & absolute & $0.6 \mathrm{~mm}$ & $9.9 \mathrm{gr}$ & $1.6 \mathrm{~mm}$ & $22.0 \mathrm{gr}$ & $2.5 \mathrm{~mm}$ & $41.6 \mathrm{gr}$ \\
\hline & improvement & $75.5 \%$ & $41.7 \%$ & $74.5 \%$ & $28.3 \%$ & $72.9 \%$ & $-1.0 \%$ \\
\hline \hline Approx. TPS & absolute & $0.6 \mathrm{~mm}$ & $9.9 \mathrm{gr}$ & $1.6 \mathrm{~mm}$ & $22.0 \mathrm{gr}$ & $2.5 \mathrm{~mm}$ & $41.6 \mathrm{gr}$ \\
\hline & improvement & $75.5 \%$ & $41.7 \%$ & $74.5 \%$ & $28.3 \%$ & $72.9 \%$ & $-1.0 \%$ \\
\hline \hline Interpol. GEBS & absolute & $0.8 \mathrm{~mm}$ & $7.5 \mathrm{gr}$ & $1.7 \mathrm{~mm}$ & $12.3 \mathrm{gr}$ & $1.9 \mathrm{~mm}$ & $33.2 \mathrm{gr}$ \\
\hline & improvement & $68.2 \%$ & $55.6 \%$ & $72.0 \%$ & $59.9 \%$ & $78.8 \%$ & $19.6 \%$ \\
\hline \hline Approx. GEBS & absolute & $0.5 \mathrm{~mm}$ & $6.8 \mathrm{gr}$ & $1.1 \mathrm{~mm}$ & $13.8 \mathrm{gr}$ & $1.3 \mathrm{~mm}$ & $28.3 \mathrm{gr}$ \\
\hline & improvement & $81.2 \%$ & $60.0 \%$ & $82.7 \%$ & $55.2 \%$ & $85.9 \%$ & $31.3 \%$ \\
\hline
\end{tabular}

images. We placed nine landmarks at the (fixed) bottom face of the cube as well as nine landmarks at the top face (see Fig. 2, right). To quantitatively determine the registration accuracy, we computed the mean geometric error $\bar{e}_{\text {geom }}$ between the 343 spheres of the (registered) source image (uncompressed cube) and the corresponding 343 spheres of the target image (compressed cube). In addition, we computed the mean intensity error $\bar{e}_{i n t}$. For the anisotropic errors we chose a value of $\sigma_{y}=1$ vox in the direction of the landmark displacements as well as $\sigma_{x z}=8 \operatorname{vox}\left(\right.$ top) and $\sigma_{x z}=3 \operatorname{vox}$ (bottom face) for the orthogonal directions.

Table 1 summarizes the registration results. Applying the new approach, the mean geometric error $\bar{e}_{\text {geom }}$ improved by $81.2 \%, 82.7 \%$, and $85.9 \%$ w.r.t. the unregistered images for strains of $5 \%, 12.5 \%$, and $18.25 \%$, respectively. In comparison, for interpolating GEBS the results are worse with improvements of $68.2 \%, 72.0 \%$, and $78.8 \%$, respectively. For interpolating TPS the results are also worse, in particular, for a strain of $18.25 \%$. Note that for approximating TPS the results did not improve in comparison to interpolating TPS, probably because TPS do not cope with cross-effects in deformations (see also below). The results based on the mean intensity error $\bar{e}_{i n t}$ are comparable with the results for $\bar{e}_{\text {geom. }}$. As an example, Fig. 3 shows 2D sections of the registered source image overlayed with the computed edges of the target image (18.25\% strain). It can clearly be seen that the compression of the cube leads to a bending at the sides 

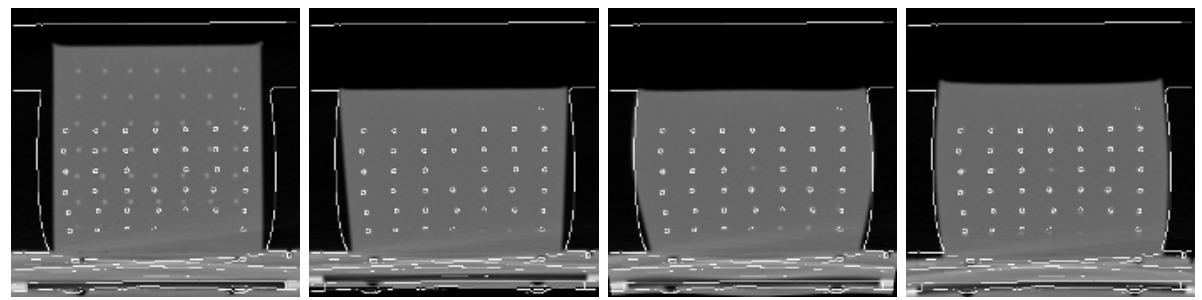

Fig. 3. Truth Cube experiment: Source image overlayed with computed edges of the target image ( $18.25 \%$ strain) without registration as well as with registration using interpolating TPS, interpolating GEBS, and approximating GEBS (from left to right)

(see the computed edges). Comparing the registration results of TPS and GEBS, it can be seen that the registered image using TPS (middle left) does not reveal a bending whereas using GEBS (middle right and right) the bending is modeled much better. The reason is that with GEBS cross-effects are taken into account, i.e. a contraction in one direction leads to a dilation in orthogonal directions.

\section{$3.2 \quad 2 \mathrm{D}$ MR Images}

We have also applied our approach to register pre- and postsurgical MR images of the human brain. Fig. 4 (left) shows 2D MR images of a patient before (source image, top) and after (target image, bottom) the resection of a tumor. 27 landmarks have been used along the contours of the tumor and the head (indicated by crosses). To simulate landmark localization in clinical routine, which is generally prone to error, we have misplaced five landmarks at the outer skull at the left side. For these misplaced landmarks, we defined the localization uncertainties in accordance with their displacements, whereas for the other landmarks we chose small isotropic uncertainties. Prior to elastic registration the images have initially been aligned by an affine transformation. Note that after affine transformation the ventricular system is well aligned, thus the resection of the tumor has only a minor effect on the position and size of the ventricular system. Fig. 4 shows the registered source image overlayed with the computed edges of the target image for interpolating GEBS (bottom middle) and approximating GEBS (bottom right). It can be seen that in both cases the vicinity of the tumor and resection area are well registered. However, it turns out that the misplaced landmarks significantly affect the registration result in the case of interpolating GEBS, see the unrealistic oscillations at the outer skull (Fig. 4, bottom middle, indicated by the arrows). In contrast, using approximating GEBS the influence of the misplaced landmarks is relative low, while the tumor and resection area are still well registered. For a comparison, we have also applied interpolating and approximating TPS (Fig. 4 top middle and right, respectively). In both cases the vicinity of the tumor and the resection area are well registered. However, there are significant errors at the ventricular system. In contrast, using GEBS only deforms the image in the vicinity of the landmarks and does not significantly affect other parts of the image. 

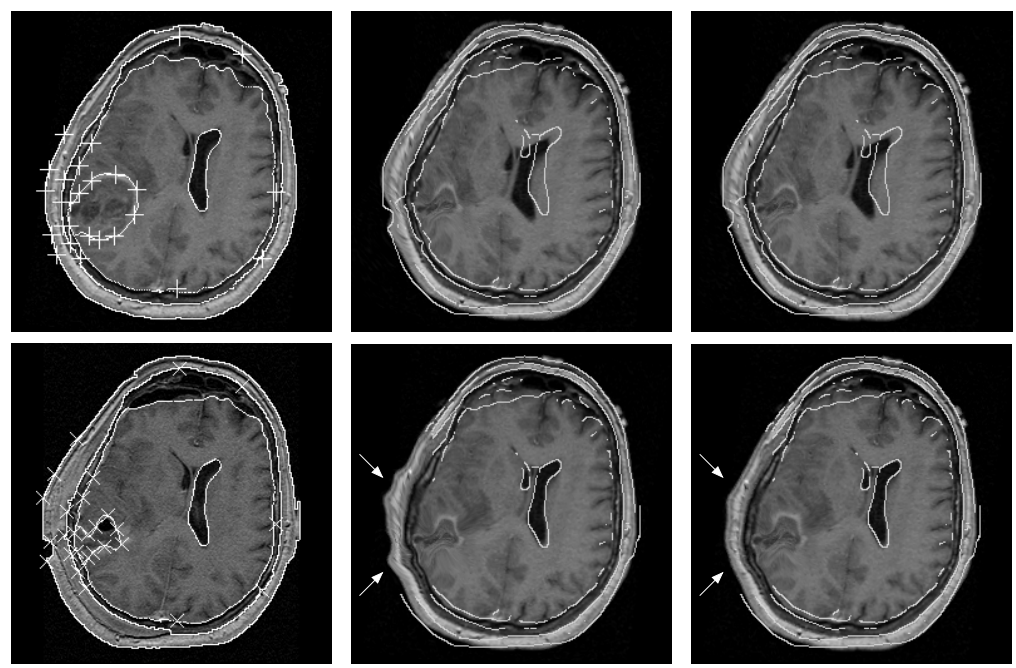

Fig. 4. Registration of $2 \mathrm{D}$ MR brain images: Pre- (top left) and postsurgical image (bottom left) as well as registered source images overlayed with computed edges of the target image using interpolating TPS (top middle), approximating TPS (top right), interpolating GEBS (bottom middle), and approximating GEBS (bottom right)

\subsection{D Gel Electrophoresis Images}

We have also applied our scheme to 2D gel electrophoresis images. As an example, Fig. 5 shows a section of a source image overlayed with computed edges of the target image (top left) and the target image overlayed with marked landmarks and computed error ellipses (bottom left). Using interpolating GEBS (bottom middle), it turns out that most corresponding spots except for the largest spot match quite well. The mean intensity error $\bar{e}_{i n t}$ improves by $17.1 \%$ w.r.t. the unregistered case. In contrast, using approximating GEBS (bottom right) yields a significantly more accurate result since also the largest spot is well matched $\left(\bar{e}_{\text {int }}\right.$ improves by $40.1 \%$ ). Interpolating and approximating TPS (top middle and top right) yield worse results with improvements of $0.8 \%$ and $35.1 \%$, respectively.

\section{Conclusion}

We have presented a new elastic registration approach, which is based on a physical deformation model, uses Gaussian elastic body splines (GEBS), and incorporates localization uncertainties of the landmarks. Since the approach is based on a physical deformation model, cross-effects in elastic deformations can be taken into account. We have demonstrated the applicability of our scheme based on different types of images. It turned out that the new approach achieves more accurate registration results in comparison to previously proposed interpolating GEBS as well as TPS. 

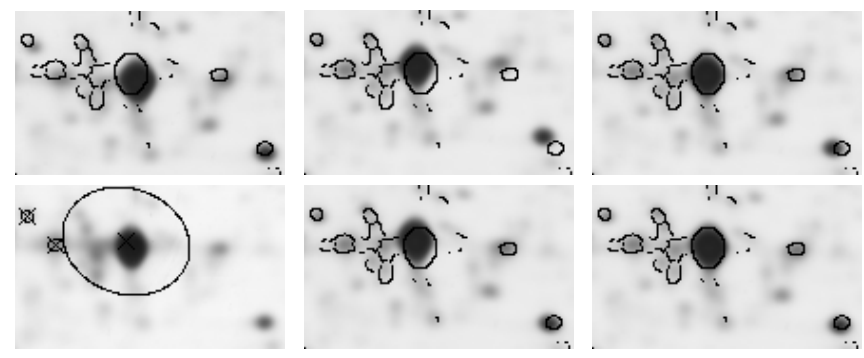

Fig. 5. Registration of 2D electrophoresis images: Source image overlayed with computed edges of the target image without registration (top left) as well as with registration using interpolating TPS (top middle), approximating TPS (top right), interpolating GEBS (bottom middle), and approximating GEBS (bottom right). Also, the target image overlayed with marked landmarks and error ellipses is shown (bottom left).

\section{Acknowledgment}

This work has been funded by the Deutsche Forschungsgemeinschaft (DFG) within the project ELASTIR (RO 2471/2-1). The original MR images and the tumor outlines have been provided by OA Dr. med. U. Spetzger and Prof. Dr. J.M. Gilsbach, Neurosurgical Clinic, University Hospital Aachen of the RWTH.

\section{References}

1. Zitova, B., Flusser, J.: Image Registration Methods: a Survey. Image and Vision Computing 24 (2003) 977-1000

2. Davis, M., Khotanzad, A., Flaming, D., Harms, S.: Physics-based coordinate transformation for 3D image matching. IEEE Trans. Med. Imag. 16 (1997) 317-328

3. Joshi, S., Miller, M.: Landmark Matching Via Large Deformation Diffeomorphisms. IEEE Trans. on Image Processing 9 (2000) 1357-1370

4. Rohr, K., Stiehl, H., Sprengel, R., Buzug, T., Weese, J., Kuhn, M.: LandmarkBased Elastic Registration Using Approximating Thin-Plate Splines. IEEE Trans. on Medical Imaging 20 (2001) 526-534

5. Kohlrausch, J., Rohr, K., Stiehl, H.: A New Class of Elastic Body Splines for Nonrigid Registration of Medical Images. In: Proc. BVM, Springer (2001) 164-168

6. Johnson, H., Christensen, G.: Consistent Landmark and Intensity-based Image Registration. IEEE Trans. on Medical Imaging 21 (2002) 450-461

7. Rohde, G., Aldroubi, A., Dawant, B.: The adaptive bases algorithm for intensity based nonrigid image registration. IEEE Trans. Med. Imag. 22 (2003) 1470-79

8. Park, H., Bland, P., Brock, K., Meyer, C.: Adaptive registration using local information measures. Medical Image Analysis 8 (2004) 465-473

9. Kohlrausch, J., Rohr, K., Stiehl, H.: A New Class of Elastic Body Splines for Non-rigid Registration of Medical Images. J. Math. Imag. Vis. 23 (2005) 253-280

10. Pekar, V., Gladilin, E., Rohr, K.: An adaptive irregular grid approach for 3-D deformable image registration. Physics in Med. and Biol. 51 (2006) 361-377

11. Kerdok, A., Cotin, S., Ottensmeyer, M., Galea, A., Howe, R., Dawson, S.: Truth cube: Establishing physical standards for soft tissue simulation. Medical Image Analysis 7 (2003) 283-291 